\title{
Dual Transformations in Galilean Spaces
}

\author{
Gülsüm Yüca* and Yusuf Yaylı \\ (Communicated by Kazım İlarslan)
}

\begin{abstract}
In this study, we define a dual transformation between $G^{n}$ and $G_{1}^{n}$. We examine the invariance of the plane where the shear motion is acting in Galilean and pseudo-Galilean spaces. We define a dual transformation between $\widehat{G^{n}}$ and $\widehat{G_{1}^{n}}$ as well. We provide applications in $G^{3}$ and $G_{1}^{3}$. In addition to applications, we draw their figures in order to reinforce the visualization in both spaces.
\end{abstract}

Keywords: dual transformation; Galilean space; pseudo-Galilean space; shear motion; one-parameter motions.

AMS Subject Classification (2020): Primary: 53A17; Secondary: 53A35; $17 B 45$.

\section{Introduction}

In [1], the geometry of motions in $G^{n}$ and $G_{1}^{n}$ was studied. Dual transformation between $S O(n) \backslash\left\{a_{n n}=0\right\}$ and $S O(n-1,1)$ was defined in [2]. In our previous paper [3], we defined a dual transformation between dual spaces of Euclidean and Lorentzian spaces. In [4], we gave a dual transformation between Lie algebras of Lie groups and draw a commutative diagram. In this study, we will give a transition between Galilean orthogonal matrices and pseudo-Galilean orthogonal matrices by defining a dual transformation between them. We investigate the invariance of the plane where the shear motion is acting in Galilean and pseudoGalilean spaces. A dual transformation between dual spaces which are $\widehat{G^{n}}$ and $\widehat{G_{1}^{n}}$ also will be defined. Moreover, we do applications and draw their figures.

First, we give basic concepts and definitions which we use during this study.

Definition 1.1. Let $X=\left(x_{1}, x_{2}, \ldots, x_{n}\right) \in \mathbb{R}^{n}$ and $G^{n}$ be Galilean space $\left(\mathbb{R}^{n},\|\|\right)$ with

$$
\|X\|=\left\{\begin{array}{cc}
\left|x_{1}\right|, & x_{1} \neq 0 \\
\sqrt{x_{2}^{2}+x_{3}^{2}+\ldots+x_{n}^{2}}, & x_{1}=0 .
\end{array}\right.
$$

Theorem 1.1. Let $A$ be an $n \times n$ matrix,

$$
A=\left[\begin{array}{c|c}
A_{1} & C \\
\hline 0 & 1
\end{array}\right]
$$

where $A_{1} \in S O(n-1)$ and $C=\left[\begin{array}{c}a_{1 n} \\ a_{2 n} \\ \vdots \\ a_{n-1 n}\end{array}\right] \in \mathbb{R}_{1}^{n-1}$.

$f$ is a Galilean transformation, where

$$
\begin{aligned}
f: G^{n} & \rightarrow G^{n} \\
X & \mapsto f(X)=A X+C .
\end{aligned}
$$


In $G^{3}$, shear movement determined with the help of $f$ with

$$
\begin{aligned}
f: G^{3} & \rightarrow G^{3} \\
X & \mapsto f(X)=A X .
\end{aligned}
$$

$f$ is also called motion in the meaning of Galilean.

Definition 1.2. Let $X=\left(x_{1}, x_{2}, \ldots, x_{n}\right) \in \mathbb{R}^{n}$ and $G_{1}^{n}$ be pseudo-Galilean space $\left(\mathbb{R}^{n},\|\|\right)$ with

$$
\|X\|_{P G}=\left\{\begin{array}{cc}
\left|x_{1}\right|, & x_{1} \neq 0 \\
\left|x_{2}{ }^{2}+x_{3}{ }^{2}+\ldots+x_{n-1}{ }^{2}-x_{n}{ }^{2}\right|, & x_{1}=0 .
\end{array}\right.
$$

In $G_{1}^{3}$ pseudo-Galilean space,

$$
\begin{aligned}
f: G_{1}^{3} & \rightarrow G_{1}^{3} \\
X & \mapsto f(X)=A X
\end{aligned}
$$

is called shear motion.

Theorem 1.2. Let $A$ be an $n \times n$ matrix,

$$
A=\left[\begin{array}{c|c}
A_{1} & C \\
\hline 0 & 1
\end{array}\right]
$$

where $A_{1} \in S O(n-2,1), \quad A_{1}{ }^{-1}=G A_{1}{ }^{T} G, \quad G^{-1}=G^{T}$ and $C=\left[\begin{array}{c}a_{1 n} \\ a_{2 n} \\ \vdots \\ a_{n-1 n}\end{array}\right] \in \mathbb{R}_{1}^{n-1}$.

$f$ is a pseudo-Galilean transformation, where

$$
\begin{aligned}
f: G_{1}^{n} & \rightarrow G_{1}^{n} \\
X & \mapsto f(X)=A X+C .
\end{aligned}
$$

See [1].

Definition 1.3. Dual transformation between $S O(n) \backslash\left\{a_{n n}=0\right\}$ and $S O(n-1,1)$ is defined in [2]. Therefore, two sets are given by

$$
\begin{gathered}
S O(n)=\left\{A \in G L(n, \mathbb{R}) \mid A^{T} A=A A^{T}=I_{n}, \operatorname{det} A=1\right\}, \\
S O(n-1,1)=\left\{A \in G L(n, \mathbb{R}) \mid A^{T} G A=A G A^{T}=G, \operatorname{det} A=1\right\},
\end{gathered}
$$

$G=\left[\begin{array}{c|c}I_{n-1} & 0 \\ \hline 0 & -1\end{array}\right]$ is the sign matrix and $I_{n}$ is $n \times n$ identity matrix.

Let $A$ be an $n \times n$ orthogonal matrix written in blocks:

$$
A=\left[\begin{array}{c|c}
B & C \\
\hline D & a_{n n}
\end{array}\right]
$$

$a_{n n} \neq 0 . B$ is $(n-1) \times(n-1)$ square matrix, $C$ is $(n-1) \times 1$ column matrix and $D$ is a row matrix. Since $a_{n n} \neq 0$, then the following two sets can be given by

$$
\begin{gathered}
X=\left\{A \in S O(n) \mid a_{n n} \neq 0\right\}, \\
Y=\left\{A \in S O(n-1,1) \mid a_{n n} \neq 0\right\} .
\end{gathered}
$$

We can define the dual transformation with

$$
\begin{aligned}
& f: X \rightarrow Y \\
& f: A \mapsto f(A)=\frac{1}{a_{n n}}\left[\begin{array}{c|c}
a_{n n}\left(B^{-1}\right)^{T} & C \\
\hline-D & 1
\end{array}\right],
\end{aligned}
$$

where $T$ is tranpose. 
Definition 1.4. If $a$ and $a^{*}$ are real numbers and $\varepsilon^{2}=0$, the combination $\widehat{a}=a+\varepsilon a^{*}$ is called a dual number, where $\varepsilon$ is dual unit.

The set of all dual numbers forms a commutative ring over the real number field and is denoted by $\mathbb{D}$.

$$
\mathbb{D}^{3}=\left\{\widehat{a}=\left(\widehat{a}_{1}, \widehat{a}_{2}, \widehat{a}_{3}\right) \mid \widehat{a}_{i} \in D, 1 \leq i \leq 3\right\}
$$

is a module over the ring $\mathbb{D}$. It is also called $\mathbb{D}$-module or dual space. The elements of $\mathbb{D}^{3}$ are called dual vectors. See [7].

Definition 1.5. We have defined $f$ dual transformation in [4]. In order to give the definition, we need to write the following sets:

$$
\begin{gathered}
S \widehat{O}(n)=\left\{\widehat{A} \in G L(n, \mathbb{D}) \mid \widehat{A}^{T} \widehat{A}=\widehat{A} \widehat{A}^{T}=I_{n}, \operatorname{det} \widehat{A}=1\right\}, \\
S \widehat{O}(n-1,1)=\left\{\widehat{A} \in G L(n, \mathbb{D}) \mid \widehat{A}^{T} G \widehat{A}=\widehat{A} G \widehat{A}^{T}=G, \operatorname{det} \widehat{A}=1\right\},
\end{gathered}
$$

$G=\left[\begin{array}{c|c}I_{n-1} & 0 \\ \hline 0 & -1\end{array}\right]$ is the sign matrix and $I_{n}$ is $n \times n$ identity matrix.

Let $\widehat{A}$ be an $n \times n$ dual orthogonal matrix $\widehat{A}$ given in blocks,

$$
\widehat{A}=\left[\begin{array}{c|c}
\widehat{B} & \widehat{C} \\
\hline D & \widehat{a}_{n n}
\end{array}\right]
$$

$\widehat{a}_{n n} \neq 0$. Since $\widehat{a}_{n n} \neq 0$, then two sets can be written as

$$
\begin{gathered}
\widehat{X}=\left\{\widehat{A} \in S \widehat{O}(n) \mid \widehat{a}_{n n} \neq 0\right\}, \\
\widehat{Y}=\left\{\widehat{A} \in S \widehat{O}(n-1,1) \mid \widehat{a}_{n n} \neq 0\right\} .
\end{gathered}
$$

$f$ dual transformation can be defined as

$$
\begin{aligned}
& f: \widehat{X} \rightarrow \widehat{Y} \\
& f: \widehat{A} \mapsto f(\widehat{A})=\frac{1}{\widehat{a}_{n n}}\left[\begin{array}{c|c}
\widehat{a}_{n n}\left(\widehat{B}^{-1}\right)^{t} & \widehat{C} \\
\hline-\widehat{D} & 1
\end{array}\right] .
\end{aligned}
$$

\section{Dual Transformation Between $G^{n}$ and $G_{1}^{n}$}

By using $f$ dual transformation which is given in [2], we obtain semi-orthogonal matrices from orthogonal matrices. In this section, we will carry the dual transformation into Galilean spaces which are $G^{n}$ and $G_{1}^{n}$.

Theorem 2.1. Let $\tilde{A} \in G^{n}$ given by

$$
\tilde{A}=\left[\begin{array}{ccc|c} 
& & a_{1 n} \\
& A & & \vdots \\
& & & a_{n-1 n} \\
\hline 0 & \ldots & 0 & 1
\end{array}\right],
$$

where $A \in S O(n-1)$.

$g$ defines a dual transformation,

$$
\begin{aligned}
& g: G^{n} \rightarrow G_{1}^{n} \\
& \tilde{A} \mapsto g(\tilde{A})=\tilde{B}=\left[\begin{array}{ccc|c} 
& & a_{1 n} \\
& f(A) & & \vdots \\
& & & a_{n-1 n} \\
\hline 0 & \ldots & 0 & 1
\end{array}\right],
\end{aligned}
$$

where $f$ is the dual transformation given in Eq. (1.5), thus $f(A) \in S O(n-2,1)$. 
Proof. $\mathrm{g}$ is a dual transformation, since it satisfies

$$
\begin{aligned}
g^{2}(\tilde{A}) & =g(g(\tilde{A})) \\
& =g(\tilde{B}), \quad f^{2}=i d . \\
& =A \\
& \Longrightarrow g^{2}=i d .
\end{aligned}
$$

Example 2.1. Let $\tilde{A}$ an $3 \times 3$ matrix given as below,

$$
\tilde{A}=\left[\begin{array}{ccc}
\frac{1}{\sqrt{2}} & -\frac{1}{\sqrt{2}} & -2 \\
\frac{1}{\sqrt{2}} & \frac{1}{\sqrt{2}} & -2 \\
0 & 0 & 1
\end{array}\right] .
$$

We apply $g$ dual transformation to Galilean matrix $\tilde{A}$, then we obtain the pseudo-Galilean matrix $g(\tilde{A})=\tilde{B}$.

$$
\tilde{B}=\left[\begin{array}{ccc}
\sqrt{2} & -1 & -2 \\
-1 & \sqrt{2} & -2 \\
0 & 0 & 1
\end{array}\right] \text {. }
$$

\section{Geometric Applications on Dual Transformation}

In this section, applications are provided with the help of $g$ dual transformation. First, we investigate the invariance of the plane where the shear motion is acting in Galilean and pseudo-Galilean spaces.

Theorem 3.1. Let $g$ is a dual transformation given by

$$
\begin{aligned}
& g: G^{n} \rightarrow G_{1}^{n} \\
& \tilde{A} \mapsto g(\tilde{A})=\tilde{B}=\left[\begin{array}{ccc|c} 
& & a_{1 n} \\
& f(A) & & \vdots \\
& & & a_{n-1 n} \\
\hline 0 & \ldots & 0 & 1
\end{array}\right],
\end{aligned}
$$

where $f$ is the dual transformation given in Eq. (1.5), thus $f(A) \in S O(n-2,1) . g$ leaves invariant the plane where the shear motion is acting.

Proof. Since the shear motion is the same in Galilean and pseudo-Galilelan space (see Eq.1.2 and Eq. 1.3), then the plane where the shear motion is acting left invariant under $g$ dual transformation.

Let us go on with examples on surfaces which are obtained by multiplying Galilean and pseudo-Galilean matrices with curves. We also draw their figures in order to observe appearances of two surfaces in both spaces.

Example 3.1. Let $\tilde{A}$ be a $3 \times 3$ Galilean matrix is given by

$$
\tilde{A}=\left[\begin{array}{ccc}
\cos \theta & -\sin \theta & 1 \\
\sin \theta & \cos \theta & 1 \\
0 & 0 & 1
\end{array}\right] .
$$

If we multiply the matrix $\tilde{A}$ and the curve $\phi(\alpha)=(\cos \alpha, 0, \sin \alpha)$ which is a circle, then we get the matrix below. 


$$
\tilde{A} \cdot \phi(\alpha)=\left[\begin{array}{ccc}
\cos \theta & -\sin \theta & 1 \\
\sin \theta & \cos \theta & 1 \\
0 & 0 & 1
\end{array}\right] \cdot\left[\begin{array}{c}
\cos \alpha \\
0 \\
\sin \alpha
\end{array}\right]=\left[\begin{array}{c}
\cos \theta \cdot \cos \alpha+\sin \alpha \\
\sin \theta \cdot \cos \alpha+\sin \alpha \\
\sin \alpha
\end{array}\right]
$$

The elements of matrix $\tilde{A} . \phi(\alpha)$ can be represented by a surface

$$
M_{1}=\Psi(\theta, \alpha)=(\cos \theta \cdot \cos \alpha+\sin \alpha, \sin \theta \cdot \cos \alpha+\sin \alpha, \sin \alpha) .
$$

See Fig. 1

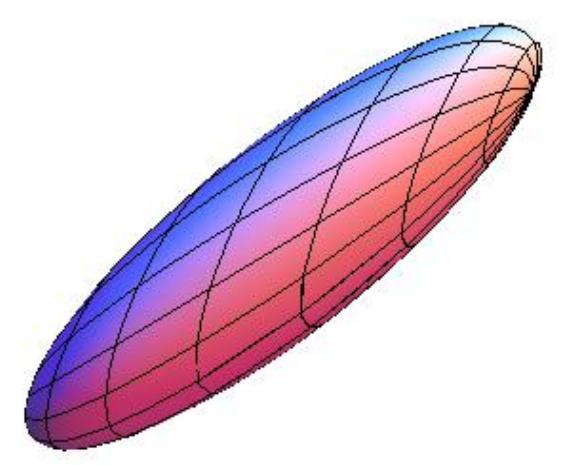

Figure 1. The surcafe $M_{1} \in G^{3}$

With the help of $g$ dual transformation, we obtain the pseudo-Galilean matrix $g(\tilde{A})=\tilde{B}$.

$$
\tilde{B}=\left[\begin{array}{ccc}
\sec \theta & -\tan \theta & 1 \\
-\tan \theta & \sec \theta & 1 \\
0 & 0 & 1
\end{array}\right] .
$$

Multiplying the matrix $\tilde{B}$ by $\phi(\alpha)=(\cos \alpha, 0, \sin \alpha)$, therefore the matrix $\tilde{B} \cdot \phi(\alpha)$ is obtained as follows,

$$
\tilde{B} . \phi(\alpha)=\left[\begin{array}{ccc}
\sec \theta & -\tan \theta & 1 \\
-\tan \theta & \sec \theta & 1 \\
0 & 0 & 1
\end{array}\right] \cdot\left[\begin{array}{c}
\cos \alpha \\
0 \\
\sin \alpha
\end{array}\right]=\left[\begin{array}{c}
\frac{\cos \alpha}{\cos \theta}+\sin \alpha \\
-\tan \theta \cdot \cos \alpha+\sin \alpha \\
\sin \alpha
\end{array}\right]
$$

The elements of matrix $\tilde{B} . \phi(\alpha)$ can be expressed as a surface

$$
M_{2}=\Psi(\theta, \alpha)=\left(\frac{\cos \alpha}{\cos \theta}+\sin \alpha,-\tan \theta \cdot \cos \alpha, \sin \alpha\right) .
$$

See Fig. 2 


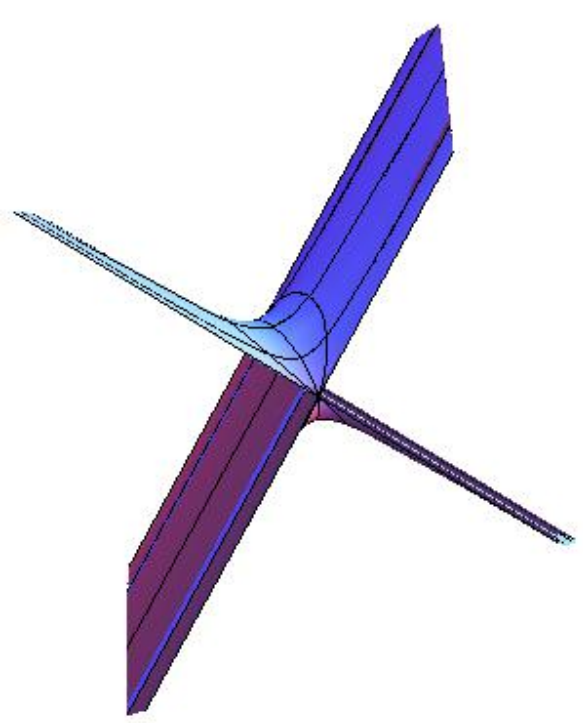

Figure 2. The surcafe $M_{2} \in G_{1}^{3}$

Example 3.2. Let us take the matrix $\tilde{A}$ from Ex. (3.1) which is a $3 \times 3$ Galilean matrix. By multiplying the matrix $\tilde{A}$ and the curve $\phi(\alpha)=\left(\frac{4}{\cos \alpha}, 0,5 \tan \alpha\right)$ which is a hyperbola, we acquire the matrix below.

$$
\tilde{A} . \phi(\alpha)=\left[\begin{array}{ccc}
\cos \theta & -\sin \theta & 1 \\
\sin \theta & \cos \theta & 1 \\
0 & 0 & 1
\end{array}\right] \cdot\left[\begin{array}{c}
\frac{4}{\cos \alpha} \\
0 \\
5 \tan \alpha
\end{array}\right]=\left[\begin{array}{c}
\frac{4 \cos \theta}{\cos \alpha}+5 \tan \alpha \\
\frac{4 \sin \theta}{\cos \alpha}+5 \tan \alpha \\
5 \tan \alpha
\end{array}\right]
$$

The elements of matrix $\tilde{A} . \phi(\alpha)$ can be represented by a surface

$$
M_{3}=\Psi(\theta, \alpha)=\left(\frac{4 \cos \theta}{\cos \alpha}+5 \tan \alpha, \frac{4 \sin \theta}{\cos \alpha}+5 \tan \alpha, 5 \tan \alpha\right)
$$

See Fig. 3

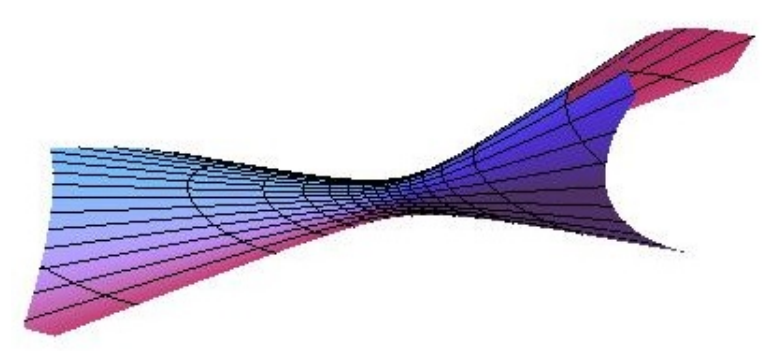

Figure 3. The surcafe $M_{3} \in G^{3}$ 
Here, we use the pseudo-Galilean matrix $\tilde{B}$ from Ex. (3.1). If we multiply $\tilde{B}$ by $\phi(\alpha)=\left(\frac{4}{\cos \alpha}, 0,5 \tan \alpha\right)$, thus the matrix $\tilde{B} . \phi(\alpha)$ is obtained as follows,

$$
\tilde{B} . \phi(\alpha)=\left[\begin{array}{ccc}
\sec \theta & -\tan \theta & 1 \\
-\tan \theta & \sec \theta & 1 \\
0 & 0 & 1
\end{array}\right] \cdot\left[\begin{array}{c}
\frac{4}{\cos \alpha} \\
0 \\
5 \tan \alpha
\end{array}\right]=\left[\begin{array}{c}
\frac{4}{\cos \theta \cdot \cos \alpha}+5 \tan \alpha \\
\frac{-4 \tan \theta}{\cos \alpha}+5 \tan \alpha \\
5 \tan \alpha
\end{array}\right] .
$$

The elements of matrix $\tilde{B} . \phi(\alpha)$ can be expressed as a surface

$$
M_{4}=\Psi(\theta, \alpha)=\left(\frac{4}{\cos \theta \cdot \cos \alpha}+5 \tan \alpha, \frac{-4 \tan \theta}{\cos \alpha}+5 \tan \alpha, 5 \tan \alpha\right) .
$$

See Fig. 4

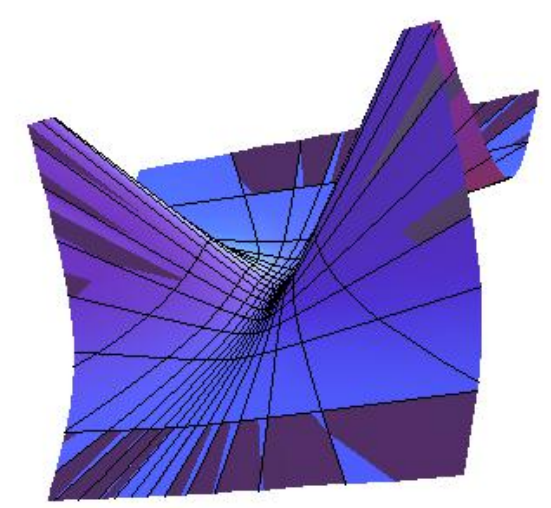

Figure 4. The surcafe $M_{4} \in G_{1}^{3}$

Example 3.3. Let us go through with an example which we take a surface. If we multiply $\phi(\theta, s)=(s, \sin s, \cos s)$ with the matrix $\tilde{A}$ from previous examples, we obtain the matrix $\tilde{A} . \phi(\theta, s)$ as below,

$$
\tilde{A} . \phi(\theta, s)=\left[\begin{array}{ccc}
\cos \theta & -\sin \theta & 1 \\
\sin \theta & \cos \theta & 1 \\
0 & 0 & 1
\end{array}\right] \cdot\left[\begin{array}{c}
s \\
\sin s \\
\cos s
\end{array}\right]=\left[\begin{array}{c}
\cos \theta \cdot s-\sin \theta \cdot \sin s+\cos s \\
\sin \theta \cdot s+\cos \theta \cdot \sin s+\cos s \\
\cos s
\end{array}\right]
$$

The elements of matrix $\tilde{A} . \phi(\theta, s)$ can be represented by a surface

$$
M_{5}=\Psi(\theta, s)=(\cos \theta \cdot s-\sin \theta \cdot \sin s+\cos s, \sin \theta \cdot s+\cos \theta \cdot \sin s+\cos s, \cos s) .
$$

See Fig. 5 


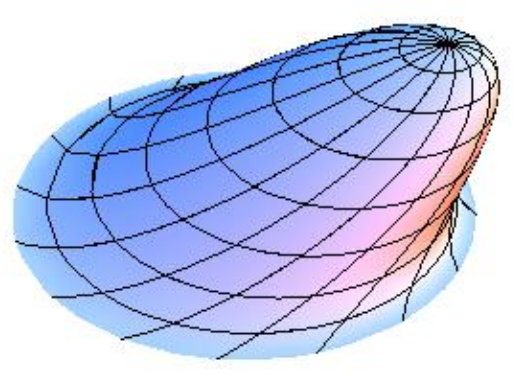

Figure 5. The surcafe $M_{5} \in G^{3}$

With the help of $g$ dual transformation, we obtain the pseudo-Galilean matrix $g(\tilde{A})=\tilde{B}$. Multiplying the matrix $\tilde{B}$ by $\phi(\theta, s)=(s, \sin s, \cos s)$, therefore the matrix $\tilde{B} . \phi(\theta, s)$ is obtained as follows,

$$
\tilde{B} . \phi(\theta, s)=\left[\begin{array}{ccc}
\sec \theta & -\tan \theta & 1 \\
-\tan \theta & \sec \theta & 1 \\
0 & 0 & 1
\end{array}\right] \cdot\left[\begin{array}{c}
s \\
\sin s \\
\cos s
\end{array}\right]=\left[\begin{array}{c}
\frac{s}{\cos \theta}-\tan \theta \cdot \sin s+\cos s \\
-\tan \theta+\frac{\sin s}{\cos \theta}+\cos s \\
\cos s
\end{array}\right] .
$$

The elements of matrix $\tilde{B} . \phi(\theta, s)$ can be expressed as a surface

$$
M_{6}=\Psi(\theta, s)=\left(\frac{s}{\cos \theta}-\tan \theta \cdot \sin s+\cos s,-\tan \theta+\frac{\sin s}{\cos \theta}+\cos s, \cos s\right) .
$$

See Fig. 6.

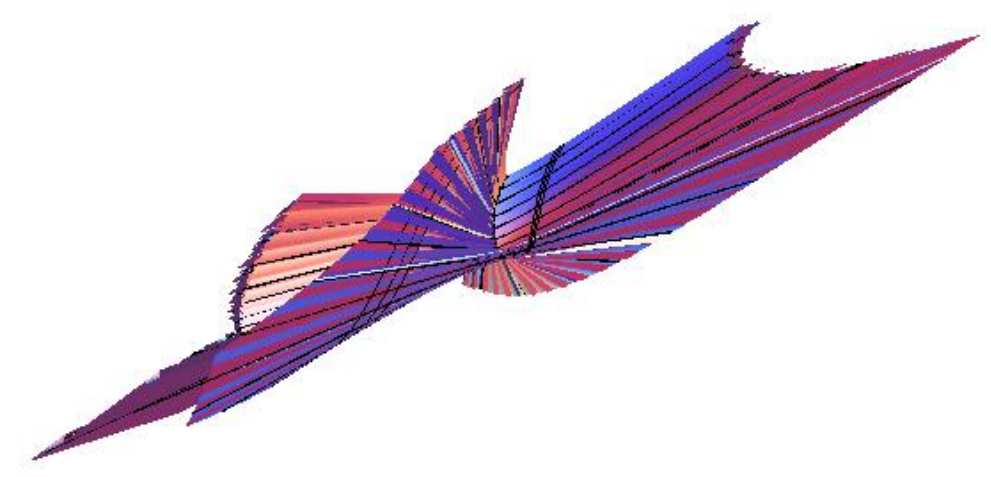

Figure 6. The surcafe $M_{6} \in G_{1}^{3}$

\section{Dual Transformation Between $\widehat{G^{n}}$ and $\widehat{G_{1}^{n}}$}

In this section, we define the dual transformation between dual Galilean spaces which are $\widehat{G^{n}}$ and $\widehat{G_{1}^{n}}$.

Theorem 4.1. Let $\widehat{\tilde{A}} \in \widehat{G^{n}}$ given by

$$
\widehat{\tilde{A}}=\left[\begin{array}{ccc|c} 
& & & \widehat{a}_{1 n} \\
& \widehat{A} & & \vdots \\
& & & \widehat{a}_{n-1 n} \\
\hline 0 & \ldots & 0 & 1
\end{array}\right]
$$


where $\widehat{A} \in S \widehat{O}(n-1)$.

$g$ defines a dual transformation,

$$
\begin{aligned}
& g: \widehat{G^{n}} \rightarrow \widehat{G_{1}^{n}} \\
& \widehat{\tilde{A}} \mapsto g(\widehat{\tilde{A}})=\widehat{\widetilde{B}}=\left[\begin{array}{ccc|c} 
& & \widehat{a}_{1 n} \\
& f(\widehat{A}) & & \vdots \\
& & & \widehat{a}_{n-1 n} \\
\hline 0 & \ldots & 0 & 1
\end{array}\right],
\end{aligned}
$$

where $f$ is the dual transformation given in Eq. (1.6), thus $f(\widehat{A}) \in S \widehat{O}(n-2,1)$.

Proof. $\mathrm{g}$ is a dual transformation, since it satisfies

$$
\begin{aligned}
g^{2}(\widehat{\tilde{A}}) & =g(g(\widehat{\tilde{A}})) \\
& =g(\widehat{\tilde{B}}), \quad f^{2}=i d . \\
& =\widehat{A} \\
& \Longrightarrow g^{2}=i d .
\end{aligned}
$$

Example 4.1. Let $\widehat{\widetilde{A}}$ an $3 \times 3$ matrix from our previous paper [3] given by

$$
\widehat{\tilde{A}}=\left[\begin{array}{cccc}
\frac{1}{\sqrt{2}}-\frac{\epsilon}{\sqrt{2}} & -\frac{1}{\sqrt{2}}-\frac{\epsilon}{\sqrt{2}} & \epsilon & -2 \\
\frac{1}{\sqrt{2}}+\frac{\epsilon}{\sqrt{2}} & \frac{1}{\sqrt{2}}-\frac{\epsilon}{\sqrt{2}} & -\epsilon & \sqrt{2} \\
0 & \frac{2 \epsilon}{\sqrt{2}} & 1 & -2 \\
0 & 0 & 0 & 1
\end{array}\right] .
$$

Under the $g$ dual transformation to Galilean matrix $\widehat{\tilde{A}}$, we obtain the pseudo-Galilean matrix $g(\widehat{\tilde{A}})=\widehat{\tilde{B}}$.

$$
\widehat{\tilde{B}}=\left[\begin{array}{cccc}
\frac{1}{\sqrt{2}}-\frac{\epsilon}{\sqrt{2}} & -\frac{1}{\sqrt{2}}-\frac{\epsilon}{\sqrt{2}} & \epsilon & -2 \\
\frac{1}{\sqrt{2}}+\frac{\epsilon}{\sqrt{2}} & \frac{1}{\sqrt{2}}-\frac{\epsilon}{\sqrt{2}} & -\epsilon & \sqrt{2} \\
0 & \frac{-2 \epsilon}{\sqrt{2}} & 1 & -2 \\
0 & 0 & 0 & 1
\end{array}\right] .
$$

\section{Conclusions}

Galilean geometry is one of the non-Euclidean geometries. In this study, we examine this geometry by defining a dual transformation. We investigate the transition between Galilean matrices and pseudo-Galilean matrices. By applying dual transformation, we examine the invariance of the plane where shear motion is acting. We also define the dual transformation in dual spaces. Moreover, we do applications in $G^{3}$ and $G_{1}^{3}$ and draw their figures. 


\section{References}

[1] Tütüncü, E. E.: The Geometry of Motions in the Galile Spaces, Phd. Thesis, Ankara University Graduate School of Natural and Applied Sciences, 2009.

[2] Dohi R., Maeda Y., Mori M., Yoshida H.: A dual transformation between $S \widehat{O}(n+1)$ and $S \widehat{O}(n, 1)$ and its geometric applications, Linear Algebra and its Applications 432: (2010), 770-776.

[3] Yüca G., Yaylı Y.: A dual transformation between $S \widehat{O}(3)$ and $S \widehat{O}(2,1)$ and its geometric applications, Proc. Natl. Acad. Sci., India, Sect. A. Phys. Sci. 88-2: (2018) 267-273.

[4] Yüca G.: Kinematics Applications of Dual Transformations, manuscript submitted for publication, 2020.

[5] López R.: Differential geometry of curves and surfaces in Lorentz-Minkowski space, International Electronic Journal of Geometry 7-1: (2014) 44-107.

[6] O'Neill B.: Semi-Riemannian Geometry, Pure and Applied Mathematics, 103,Academic Press, Inc. [Harcourt Brace Jovanovich, Publishers], New York (1983).

[7] Yaylı Y., Çalışkan A., Uğurlu H.H.: The E. Study maps of circles on dual hyperbolic and Lorentzian unit spheres $H_{0}^{2}$ and $S_{1}^{2}$, Math. Proc. R. Ir. Acad. 102A-1: (2002) 37-47.

\section{Affiliations}

GÜLSÜM YÜCA

AdDress: Aksaray University, Dept. of Mathematics, 68100, Aksaray-Turkey.

E-MAIL: gulsumbicer@gmail.com

ORCID ID:0000-0002-2015-7350

YUSUF YAYLI

AdDRESS: Ankara University, Dept. of Mathematics, 06100, Ankara-Turkey.

E-MAIL: yayli@science.ankara.edu.tr

ORCID ID:0000-0003-4398-3855 